\title{
5G Embedded Sensor Network System for Sports Information Service Hotspot Recommendation
}

\author{
Zhong $\mathrm{Wu}^{1}$ and Chuan Zhou $\mathbb{D}^{2}$ \\ ${ }^{1}$ Physical Education School, Wuhan Business University, Wuhan, 430056 Hubei, China \\ ${ }^{2}$ Institute of Mechanical Engineering, Wuhan Institute of Shipbuilding Technology, Wuhan, 430062 Hubei, China \\ Correspondence should be addressed to Chuan Zhou; 20150459@wbu.edu.cn
}

Received 29 November 2021; Revised 18 January 2022; Accepted 11 February 2022; Published 4 March 2022

Academic Editor: Mohamed Elhoseny

Copyright (C) 2022 Zhong Wu and Chuan Zhou. This is an open access article distributed under the Creative Commons Attribution License, which permits unrestricted use, distribution, and reproduction in any medium, provided the original work is properly cited.

\begin{abstract}
How to rationally allocate and integrate existing resources has become the top priority of the development of sports public information services in the transition period, but it is also a weak link in the construction of current social information resources. This article is aimed at studying how to develop sports information service hotspots based on $5 \mathrm{G}$ embedded sensor network systems. Methods such as the network structure based on 5G embedded sensors, the Kriging algorithm based on global optimization, and sensor distance measurement are proposed and also conducted experiments on the application of $5 \mathrm{G}$ embedded sensor network system in sports information service hotspot recommendation. The results show that people are satisfied with the sports information service of the wireless sensor network, with a maximum score of 9.8 , which is suitable for the information provision of sports information services. The development of the times has met the needs of most people.
\end{abstract}

\section{Introduction}

With the rapid iteration of information flow in the era of big data and the professional development of the sports industry market, the information value of the sports industry has exploded, and the sports information industry has emerged as the times require. With the development of the socialist market economy, the social structure has undergone tremendous changes, and the sports management system has also undergone a fundamental change to some extent. The wave of reform and opening up and the process of economic system transformation have shaken the foundation of the original sports management system and to a large extent led its epoch-making pace. As one of the important areas of the Internet, sensor networks play an important supporting role. The sensor network is responsible for the collection and transmission of data. The sensor network is composed of multiple sensor nodes, which send the acquired data to users. Through wireless multihop communication, the acquired data is transmitted to the user terminal.

In the era of "Internet +", with more and more forms of nationwide fitness information services, based on Internet technology, the speed of software and hardware upgrades should also be accelerated, and many information service platforms with strong practical value have been established. It provides users with various services such as physical guidance, health consultation, and classes, which greatly promotes users' sports and fitness activities. This plays a very important role in promoting the healthy development of the people.

With the continuous acceleration of the development of social network informatization, sports services are becoming more and more informatized. Xu et al. believes that the urban sports energy-saving information service network has not yet formed. The rapid flow of information and sharing features has not yet been released. According to the characteristics and laws of urban sports development, the integrated functions of urban sports information network services are manifested in the aspects of universal value, cooperative organization, and diversified benefits. It is also necessary to implement the content, characteristics, and regional innovation and development strategies of sports information network services, using the reform of the 
student union portal as a driving force to strengthen the integrity and regional characteristics of urban sports information, establishing an information exchange and sharing operation platform supported by network technology, and strengthening the exchange of cooperation among various social forces. In order to explore the relationship between value balance and realization, it is possible to conduct a comprehensive investigation of the internal components of the service in combination with the new ideas developed in the era of the "smart city" service information platform, to promote the development of service information platform and design ideas and direction information platform. It can fully characterize the intelligent and humane ecological development and design concepts [1]. The purpose of Howard MS's experiment is to determine whether athletes are interested in information services and to determine whether pharmacists popularize and promote knowledge of athletes. He conducted an experimental survey of local sports athletes and collected the age, gender, and the degree of understanding of the information services of the sports athletes, including where they live. The statistical information of the sports athletes collected by his survey includes information services Interest, desire for knowledge, and obstacles to learning. The results show that their understanding of information services is not high [2]. Cain et al. has conducted research on two sports universities to understand the value of information services provided by the society and the needs of sports personnel for information services. The results show that the methods used by the two sports universities to apply sports information services depend on the needs of sports athletes. The results provide a method for the development of sports information services and help predict the changing information needs of a wider range of sports players [3]. With the popularization and development of the Internet, information has changed people's lives and production extensively and deeply, and information construction has become the main direction of sports information services in the future. In the era of rapid development of information technology, it is of great significance to study the combination of the sports industry and the information industry and evaluate the capabilities of smart sports information services. The purpose of this research is to explore a methodological approach. Evaluation of sports information services provides insights for the theoretical research and practical development. First, determine the objects and key evaluation items of intelligent sports information services through factor analysis. Secondly, the application of analytic hierarchy process takes the evaluation of sports university as an example to verify the authenticity and operability of the index system and proposes corresponding measures. The research results show that only by improving the public sports database, big data can analyze the establishment of the system and its various forms of application [4]. Gope and Hwang found that the emergence of the Internet of things is due to the continuous development of information technology. In modern sports, the use of Internet of things technology can bring convenience to sports lovers, and they are also used in various sports. Sensor network technology is one of the important factors in the development of sports information service. In this article, he found that the security function of the sports system can be met by the sports information service of the Internet of things [5]. Abraham and Li found that the indoor air quality information system helps to improve indoor air quality. In this paper, he proposed a low-cost and high-quality wireless sensor network system for indoor air quality monitoring. He also found that sensor calibration and measurement data conversion can be solved by methods based on linear least squares estimation. In this article, he introduces the theoretical knowledge and practical methods of wireless sensor nodes. Through the analysis of results and experiments, the importance of wireless sensor networks is proved [6]. Hammoudeh et al. analyze that national security is affected by external border monitoring. Wireless sensor networks can reduce costs and give solutions based on intelligent technology to effectively monitor complex areas. In this article, he uses an appropriate index to measure the quality of transmission detection. He also proposed a method to calculate the number of sensor nodes and then found a new crosslayer routing protocol, hierarchical partition graph, which is specially used to solve the communication requirements of topological linear applications [7]. Zhang et al. studied the energy-saving distributed filtering in sensor networks and found that the switching system method can achieve this goal. The first mock exam is to switch the system, and he put forward a unified model to capture the problems of low speed and poor signal. The system has obtained a sufficient condition for the exponential stability of the filtering error system in the mean square sense, and the performance of the system is qualified. All experimental studies have proved that the new design technology he proposed is very reliable [8]. Through the experiments and research of scholars, it can be known that the application of sensor networks in real life is becoming more and more extensive. The development of sports information services based on the $5 \mathrm{G}$ embedded sensor network system is a major trend today. How to use the sensor network system is a difficult problem for sports information services today.

The innovations of this article are as follows: (1) investigation and research of modern sports enthusiasts' sports information services based on wireless sensor networks through investigation and research methods, researching and analyzing the actual effects of sports applications on sports enthusiasts and then discussing how to use sports information services healthily, and (2) adopting the Kriging algorithm based on global optimization and developing sports information services based on wireless sensor networks to promote the popularization of the importance of sports information services.

\section{Kriging Algorithm Based on Global Optimization}

2.1. The Network Structure of the Sensor. Kriging is a regression algorithm for spatial modeling and prediction of stochastic processes based on covariance functions. In certain stochastic processes, such as inherently stationary processes, Kriging can give optimal linear unbiased estimates, so it is also called spatially optimal unbiased estimator in 
geostatistics. The wireless sensor network integrating sensor technology, embedded computing technology, distributed information processing technology, wireless communication technology, and the latest microelectronic technology are a new information acquisition and information processing network technology $[9,10]$. They have a wide range of application prospects and are suitable for all aspects of human activities such as national security, military, environmental monitoring, health care, and smart homes. The wireless sensor network is composed of three parts, mainly sensor nodes, base station nodes, and background user management interfaces in the field of network monitoring, as shown in Figure 1.

As shown in Figure 1, the sensor network node not only completes the collection of surrounding environmental parameters but also maintains the network topology and routing information. This is a microembedded system that can complete the fusion processing of the collected data and the data sent by other nodes $[11,12]$. Routing algorithms, also known as routing algorithms, can be differentiated by a number of characteristics. The goal of the algorithm is to find a "good" path from the source router to the destination router. At the same time, it cooperates with other nodes to complete specific tasks such as target tracking. The base station node has completed the overall network data fusion processing and conversion based on the Internet communication protocol, and its communication capabilities, processing capabilities, and storage capabilities are relatively strong [13]. User management completes tasks such as task start, data analysis, network management, and other functions.

Currently, wireless sensor networks generally adopt topological structures, and the mesh structure topology is shown in Figure 2.

As shown in Figure 2, the mesh structure is a peer-topeer structure. The nodes of all structures are sensor nodes and synchronization nodes. Nodes in the network can communicate directly when they are within the communication range. In addition, the nodes within the communication range [14] can be used as transition nodes. The characteristic of this structure is the robustness and reliability of the network, but this kind of network structure is complex and requires higher additional overhead [15].

In different applications, the composition of sensor network nodes is not the same $[16,17]$. But generally, it is composed of data acquisition unit, data processing unit, data storage unit, data transmission unit, power supply, and embedded operating system, as shown in Figure 3.

As shown in Figure 3, as a complete microcomputer system, the performance of its components must be coordinated and efficient. The choice of each module's implementation technology needs to be weighed and selected according to the actual application system requirements. The remote wireless upgrade of the wireless sensor network is a process of distributing the new code image to each node through the network to complete the replacement of the old and new codes, thereby realizing the upgrade process. It mainly includes two processes: the distribution of new application code images and the replacement of new and old codes [18].
2.2. Kriging Algorithm Based on Global Optimization. The Kriging method is based on a series of known observation points $Z_{i}=Z\left(a_{i}\right), i=1, \cdots, n$ in a random area $Z(a)$ and interpolating the function value $Z(a)$ of $a_{0}$ at the unknown point. The Kriging method is based on space, relying on observation points to quantify the variance change $\gamma(a, b)$ or expectation $\mu(a)$ and the covariance function $C(a)$ of the random model, and calculates $Z(a)$ the optimal linear unbiased estimate $Z\left(a_{0}\right)$ as formula (1):

$$
Z\left(a_{0}\right)=\sum_{i=1}^{n} \omega_{i}\left(a_{0}\right) Z\left(a_{i}\right)
$$

This type of task is usually issued by the sink node, and the sensor node collects the data that meets the requirements and reports it to the sink node [19]. The error obtained in the Kriging model is as formula (2):

$$
a_{K}^{2}\left(a_{0}\right)=\operatorname{Var}\left[Z\left(a_{0}\right)-Z\left(b_{0}\right)\right]
$$

The routing algorithm should be easy to implement and low in computational complexity. In the case of nodes with limited computing $Z\left(b_{0}\right)$ power in wireless sensor networks, it is necessary to avoid the use of complex optimization methods [20, 21]. Although multiobjective optimization algorithms exist, more single-metric optimization algorithms are used. According to the unbiased condition, formula (3),

$$
E[Z(a)-Z(b)]=\sum_{i=1}^{n} \omega_{i}\left(a_{0}\right) \mu\left(a_{i}\right)-\mu\left(a_{0}\right)=0 .
$$

First assume that the response value at point $\mathrm{A}$ to be evaluated $b(a)$ is Equation (4):

$$
b(a)=C^{T}(a) B, C^{T}(a) \in R^{n} .
$$

Under this condition, the predicted mean square error $\psi(a)$ is formula (5):

$$
\psi(a)=E\left[b(a)-b(a)^{2}\right]=E\left[C^{T} Z-Z\right]
$$

2.3. Correlation Function Algorithm. For stochastic processes, as described in formulas (4) and (5) above, the main research object is correlation function $R(\theta, \omega, a)$. The basic form of the correlation function model used in this article is formula (6);

$$
R(\theta, \omega, a)=\prod_{i=1}^{n} R\left(\theta_{i}, \omega_{i}, a_{i}\right)
$$

The "variance" $\theta_{i}$ is a measure used in probability theory to measure the degree of deviation between a random variable $\omega_{i}$ and its estimated value $a_{i}$.

The Gaussian function is named after the great mathematician Johann Karl Friedrich Gauss. The Gaussian function has a wide range of applications and can be seen in 


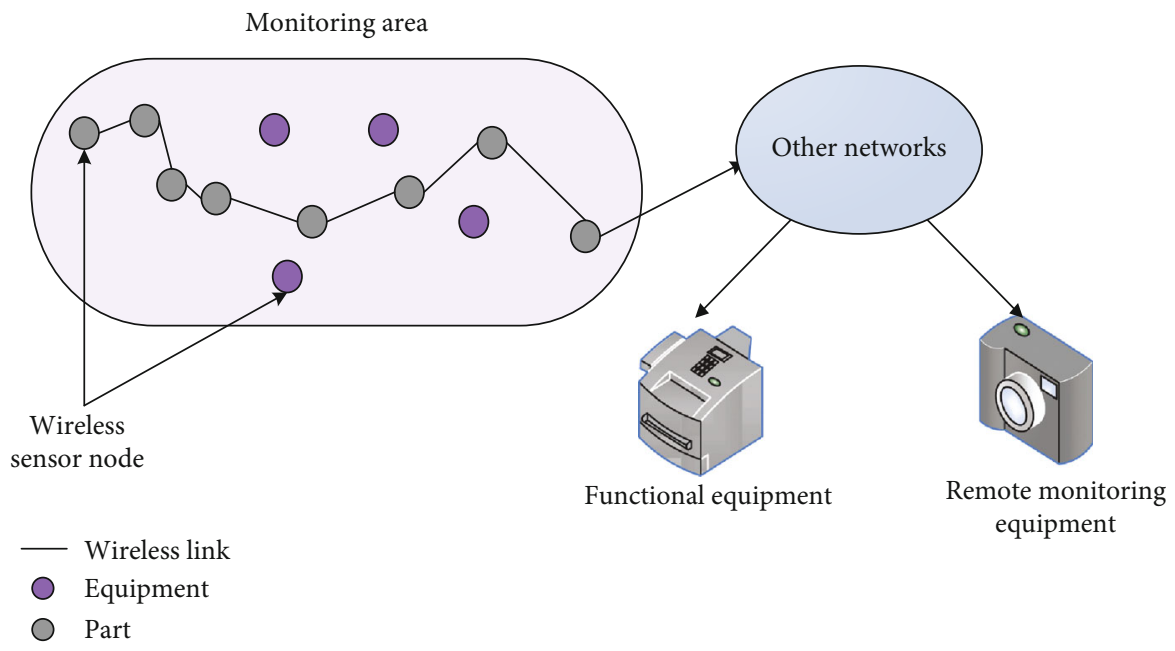

FIGURE 1: Node diagram of sensor network.

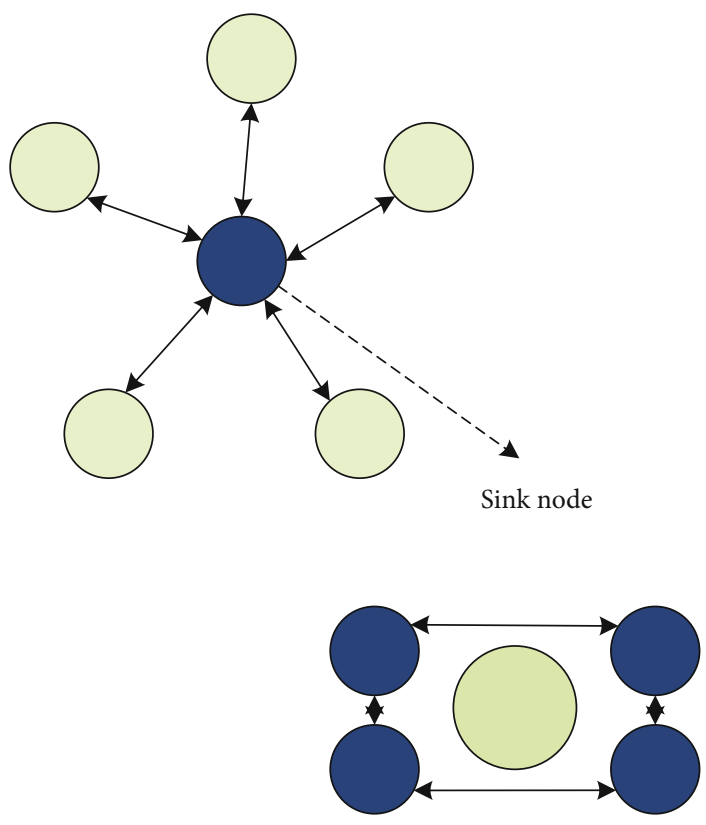

(a)

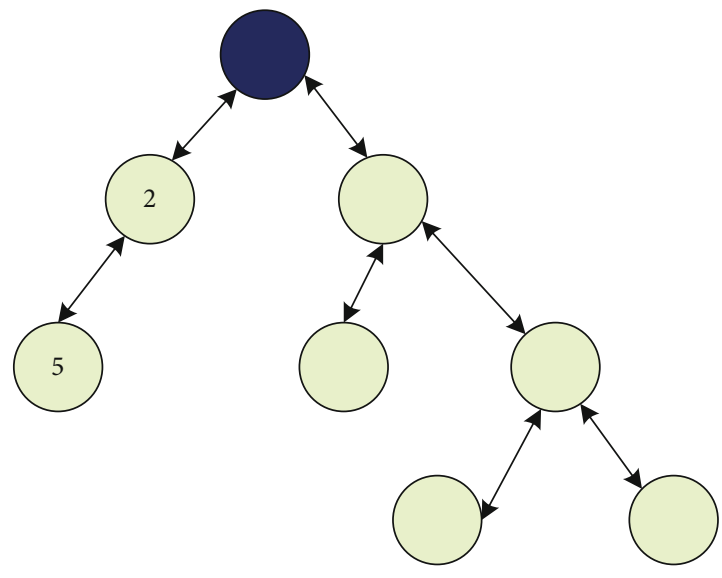

(b)

FIgURE 2: Topology diagram of mesh structure.

the natural sciences, social sciences, mathematics, and engineering. The following lists 8 different forms of correlation functions as shown in Table 1:

As shown in Table 1, the selection of correlation functions should be based on actual physical phenomena. If the phenomenon under study is continuously differentiable, then the correlation function is likely to show a parabolic linear state near the origin. That means Gaussian function, cubic function, and curve function should be selected [22]. In addition, linear functions, cubic functions, spherical functions, and curvilinear functions are compact functions. For distances greater than the support radius, its value is 0 , while other functions are global functions and tend to be asymptotically to 0 (18).
Assuming that the correlation function is a Gaussian process $\psi(\theta)$, the optimal coefficients of the correlation function can be solved, and the equation is as Equation (7):

$$
\min \left\{\psi(\theta)=\frac{1}{m} \sigma^{2}\right\}
$$

The typical trend of the expression function in Table 1 is shown in Figure 4.

As shown in Figure 4, the expression function decreases with the increase of the number of experiments, from 0.4 to 0.2 . 


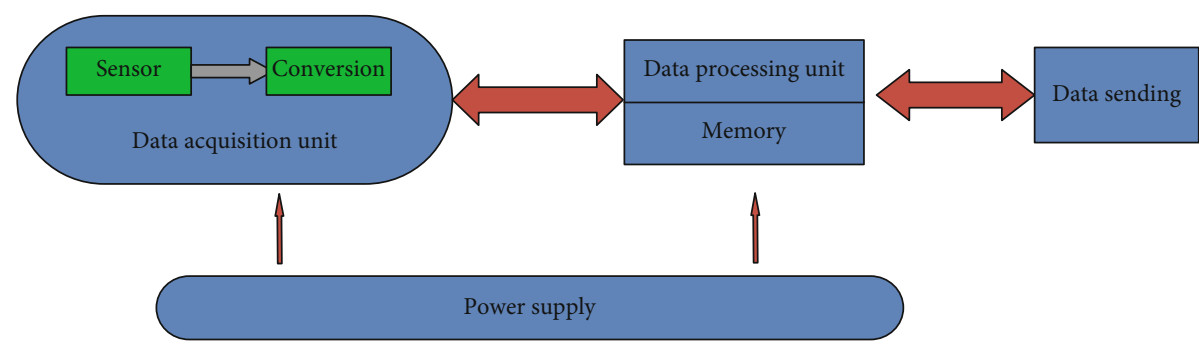

FIgURE 3: The basic composition diagram of the node.

TABLE 1: Forms of 8 different correlation functions.

\begin{tabular}{lc}
\hline Name & $R(\theta, \omega, a)$ \\
\hline EXP model & $b(a)=C^{T}(a) B, C^{T}(a) \in R^{n}$ \\
EXP model A & $b(a)=C^{T}(a) B, C^{T}(a) \in R^{n}$ \\
EXP model B & $a_{K}^{2}\left(a_{0}\right)=\operatorname{Var}\left[Z\left(a_{0}\right)-Z\left(b_{0}\right)\right]$ \\
Linear model & $Z\left(a_{0}\right)=\sum_{i=1}^{n} \omega_{i}\left(a_{0}\right) Z\left(a_{i}\right)$ \\
Gaussian model & $Z_{i}=Z\left(a_{i}\right), i=1, \cdots, n$ \\
Spline model & $\gamma(a, b)$ \\
Ball model & $R(\theta, \omega, a)=\prod_{i=1}^{n} R\left(\theta_{i}, \omega_{i}, a_{i}\right)$ \\
\hline
\end{tabular}
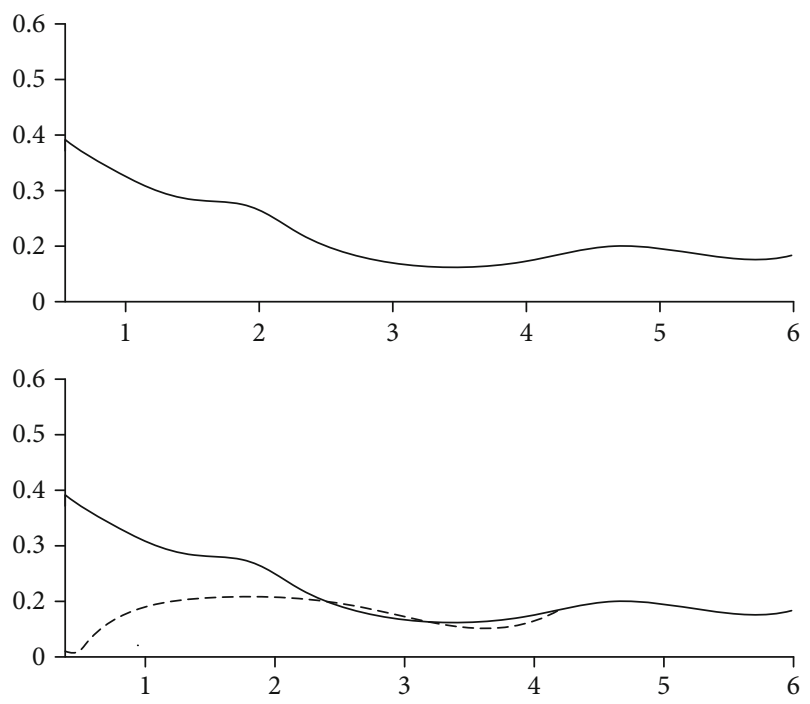

Figure 4: Typical trend chart.

2.4. Distance Measurement Method. There is an important concept in greedy embedding-distance. The distance is a function defined in the metric space. In general distance space, the distance between two points is usually measured by the norm of a vector composed of two points. In daily life, the most general distance is the distance in Euclidean space as the quadratic norm [23]. In graph theory, distance is the number of edges that pass the shortest path between two nodes. In analytic geometry, distance is a first-order norm. In the $n$-dimensional Euclidean space, there are three commonly used distance measurement methods [24].
(1) Manhattan distance (L1 distance): it is a geometric term used in Euclidean geometric metric space to indicate the sum of absolute wheelbases of two points in a standard $n$-dimensional coordinate system. The Manhattan distance of two points $a_{i}, a_{j}$ in $N$-dimensional Euclidean space is formula (8):

$$
d_{i j}=\sum_{k=1}^{n}\left|a_{i k}-a_{j k}\right| .
$$

(2) Euclidean distance (L2 distance): the most commonly used distance measurement method is also the default distance measurement method in reality. The Euclidean distance of two points $a_{i}, a_{j}$ in $N$ -dimensional Euclidean space is formula (9):

$$
d_{i j}=\sqrt{\sum_{k=1}^{n}\left|a_{i k}-a_{j k}\right|} .
$$

(3) Chebyshev distance ( $\mathrm{L}$ distance) is also called the maximum distance. It is used to indicate the maximum absolute wheelbase of two points in the standard $n$-dimensional coordinate system. The Chebyshev distance of two points $a_{i}, a_{j}$ in $N$ -dimensional Euclidean space is formula (10):

$$
d_{i j}=\max \sum_{k=1}^{n}\left|a_{i k}-a_{j k}\right| .
$$

Distance measurement is required in work such as triangulation, traverse, topographic, and engineering surveys. The accuracy of distance measurement is expressed as relative error. It can be seen from formula (10) that in order to realize the concise and greedy embedding of a graph $\sum_{k=1}^{n}\left|a_{i k}-a_{j k}\right|$, it is necessary to consider factors such as the measurement space, the distance measurement method, and the coordinate representation method. These factors provide directions for subsequent research [25], as shown in Figure 5. 


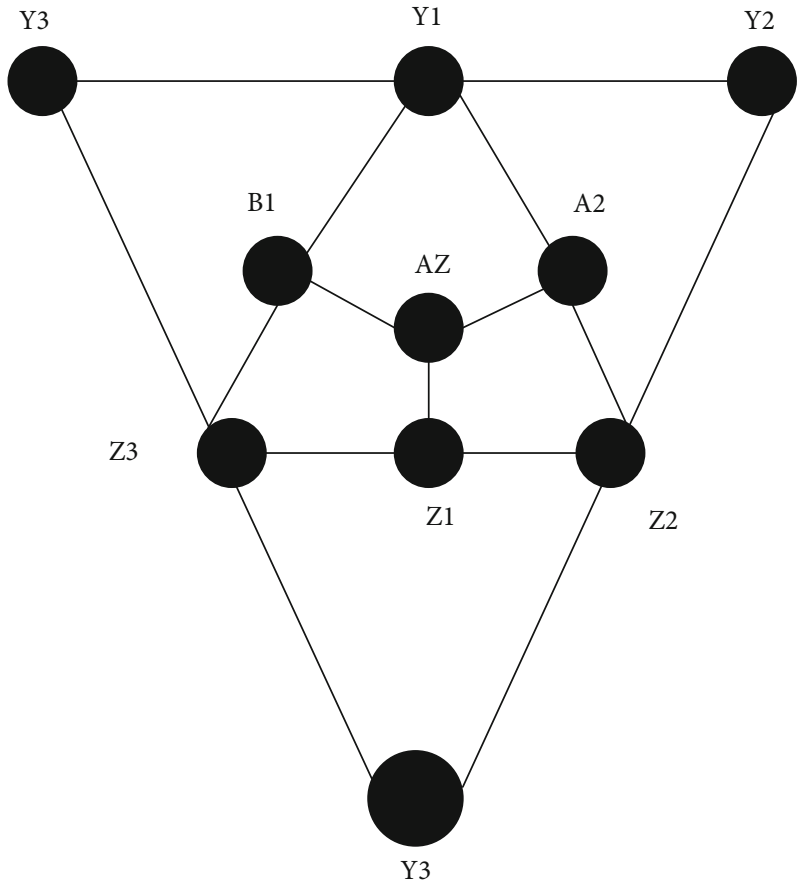

Figure 5: Recursive planar graph not greedy embedded.

As shown in Figure 5, in order to use graph theory to study wireless sensor networks, it must first determine the connectivity of the network. Through simulation experiments, the relationship between the average connectivity of the network formed by several random throws and the number of nodes in the network and the communication radius of the nodes is given [26-28].

2.5. Kriging Algorithm Implementation. For the convenience of explanation, this section uses the function $y$ to represent the above functions and constructs a Kriging model [29]. Obtaining the pressure value of a set of pressure measurement points from the sample $P_{1}, P_{2}, P_{3}, \cdots P_{N}$, its estimated value is as shown in formula (11):

$$
Y(P)=\mu(P)+Z(P) .
$$

Among them, $\mu$ is an unknown constant, $Z$ is a Gaussian random process, the expected value is zero, and the variance value is $\sigma^{2}$. The value of $\mu$ can be calculated by the least square method using the previous input data, which is formula (12):

$$
\mu=\frac{1^{T}(\varphi+\lambda I)^{-1} b}{1^{T}(\varphi+\lambda I)^{-1} 1} .
$$

Introduced by the basic principles of the Kriging algorithm in the previous section $(\varphi+\lambda I)^{-1} b$, the Kriging model can be transformed into formula (13):

$$
\phi_{i j}=\mu+\varphi^{t}(b+1) .
$$

Among them, $\varphi$ is the covariance vector, which can be

\begin{tabular}{|c|c|c|c|}
\hline Classification & State & Symbol & $\begin{array}{l}\text { Typical energy } \\
\text { consumption }\end{array}$ \\
\hline $\mathrm{CPU}$ & Start up & ECPU & $5.4 \mathrm{~mA}$ \\
\hline $\mathrm{CPU}$ & $\begin{array}{l}\text { Energy } \\
\text { saving }\end{array}$ & ECPU & $0.542 \mathrm{~mA}$ \\
\hline $\begin{array}{l}\mathrm{RF} \\
\text { transceiver }\end{array}$ & Send & ETRX & $21 \mathrm{~mA}$ \\
\hline $\begin{array}{l}\mathrm{RF} \\
\text { transceiver }\end{array}$ & Take over & ETRX & $54 \mathrm{~mA}$ \\
\hline $\begin{array}{l}\mathrm{RF} \\
\text { transceiver }\end{array}$ & Idle & ETRX & $1.8 \mathrm{~mA}$ \\
\hline $\begin{array}{l}\text { RF } \\
\text { transceiver }\end{array}$ & Dormant & ETRX & $0.07 \mathrm{~mA}$ \\
\hline
\end{tabular}
expressed as formula (14):
TABle 2: List of node energy consumption.

$$
\varphi_{i j}=\exp \left(-\sum_{k=1}^{d} \theta\left|\rho_{i k}-\rho_{j k}\right|^{i k}\right) .
$$

Among them, the value of hyperparameter $\theta$ can be used to adjust the width of the function model, and the hyperparameter can be used to adjust the smoothness of the function. The entire covariance matrix is Equation (15):

$$
\varphi_{i j}+\delta_{i j} \lambda=\exp \left(\sum_{k=1}^{d} \theta_{k}\right)^{2}
$$

The above is the Kriging model established for atmospheric data $\delta_{i j} \lambda$, but the hyperparameters need to be obtained by the maximum likelihood estimation method to facilitate calculation [30]. The maximum likelihood function of $B$ is Equation (16):

$$
L=-\frac{1}{2} n\left(\partial^{2}\right)^{2}
$$

At this point, the angle of attack and the angle of sideslip can be directly calculated through the known pressure values of each pressure measurement point $\left(\partial^{2}\right)^{2}$. And through software calculations and wind tunnel experiments, a large amount of data was obtained, and the covariance matrix was established [31].

2.6. Node Energy Consumption Analysis. The energy consumption of sensor nodes is mainly divided into two parts, namely, the energy consumption of the on-board microprocessor and the energy consumption of the radio frequency transceiver. Typical energy consumption data of sensor nodes is shown in Table 2.

As shown in Table 2, for any time slot, according to the previous assumptions, each node must be in one of the following three states: packet sending, vacancy including back-off, and interframe idle. The probabilities are, respectively, formula (17):

$$
\rho^{7}=\rho r=\text { Lpsenspcca }
$$




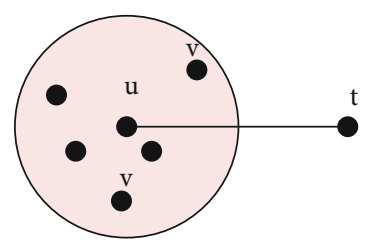

FIgURE 6: The closest rule to the destination.

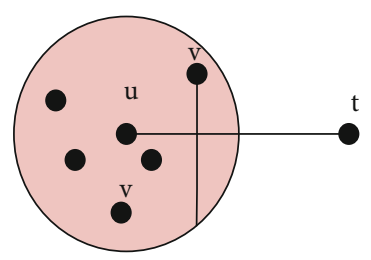

Figure 7: Maximum forward rule within a circle.

Sensor nodes are usually small embedded systems with weak processing, storage, and communication functions, and their energy is supplied by limited batteries. From the point of view of network function, each sensor node considers the dual functions of the terminal and router of the previous network node. In addition to collecting local information and processing data, it is also necessary to save, manage, and merge the transmitted data, according to other nodes cooperate with other nodes to complete several specific tasks.

The rule of closest to the destination is as follows: the current node chooses the node closest to the destination node among the neighbor nodes as the next hop node, as shown in Figure 6.

As shown in Figure 6, only the nodes closer to the destination node $t$ than the node $u$ are candidate nodes. Otherwise, it will not be able to move forward, and the algorithm will fail at this time. The line segment from $u$ to this neighboring node has the longest projection length on the line segment ut, as shown in Figure 7.

As shown in Figure 7, the nearest forward rule, a node selects the nearest neighbor node with a forward progress component as the next hop node. Node $u$ chooses node $v$ as the next hop node.

The tracking algorithm is based on the target's motion model. If the sensor node sends measurement data to the central node control node in the form of a nonfixed period, this increases the complexity of the central node's current position estimation and future position prediction algorithms for the target, which is not conducive to effective tracking. Considering the following dynamic objects, including a discrete event dynamic subsystem $a_{i}[K+1]$ as Equation (18),

$$
a_{i}[K+1]=A_{i}[k]+\omega_{i}[k] .
$$

Corresponding to each subsystem $\omega_{i}[k]$, a sensor node is deployed in a reasonable location to measure the status of each subsystem separately. The sensor measurement value $b_{i}[k]$ is Equation (19):

$$
b_{i}[k]=C_{i}[k] b[k]+u_{i}[k] .
$$

Therefore, in practical applications, the selection of the above parameters should be determined according to the network scale, protocol, environmental interference, controller calculation performance, and algorithm complexity. The centralized method has poor scalability and is not applicable when the network scale is large. With the development of hardware and emerging application requirements, control systems for large-scale networks have gradually become a trend; so, scalability has become particularly important. For this reason, the control tasks are concentrated into decentralized and fully utilized resources. The tasks of the centralized controller are decomposed and shared by some nodes, such as distributed controllers or algorithm agents, to reduce the workload of a single point, thereby improving scalability.

With the accelerated pace of work and increased life pressure in modern society, the popularization of sports and fitness information services on the Internet undoubtedly provides people with a good way to solve health problems. However, there are a series of problems such as complex network environment, explosive sports fitness information, and uneven network fitness guidance team. This brings many challenges to the sports and fitness safety of ordinary people.

\section{Experiment and Analysis Based on Sensor Network and Questionnaire}

With the development of social economy, the physical fitness of the masses has also been steadily improved. The main task of the development of information services is to promote sports culture and promote the all-round development of people. The national level attaches great importance to the physical exercise of the masses, and the masses should also actively participate in physical exercises. At present, national fitness has become an upsurge, and people's fitness awareness is slowly being awakened. Therefore, the masses' demand for sports information service information is increasing.

Information exchange and processing on the Internet prepare for the organization and arrangement of actual physical activities. Through the Internet or community service information systems, residents can enjoy online sports and fitness information services provided by the community, and realize online competition to open the time and place of sports games and activities, and information such as operating platforms provided by local residents. This article investigates the degree of satisfaction of sports fans with information services, as shown in Table 3:

As shown in Table 3, sports enthusiasts are generally satisfied with sports information services accounted for a larger proportion, very satisfied at the least, accounting for only $5.6 \%$, dissatisfied and relatively dissatisfied accounted for $16.5 \%$. It shows that the masses are very dissatisfied with the current sports information services, and there is a problem of information lag in sports information services. This 
TABLE 3: Satisfaction of sports enthusiasts with information services.

\begin{tabular}{lcccc}
\hline & Frequency & Percentage & Effective percentage & Cumulative percentage \\
\hline Very satisfied & 26 & 5.6 & 5.6 & 5.6 \\
Quite satisfied & 76 & 21.6 & 21.6 & 30 \\
Generally & 154 & 52.5 & 52.5 & 89 \\
Relatively dissatisfied & 34 & 6.7 & 6.7 & 76 \\
Dissatisfied & 20 & 7.8 & 7.8 & 78 \\
\hline
\end{tabular}

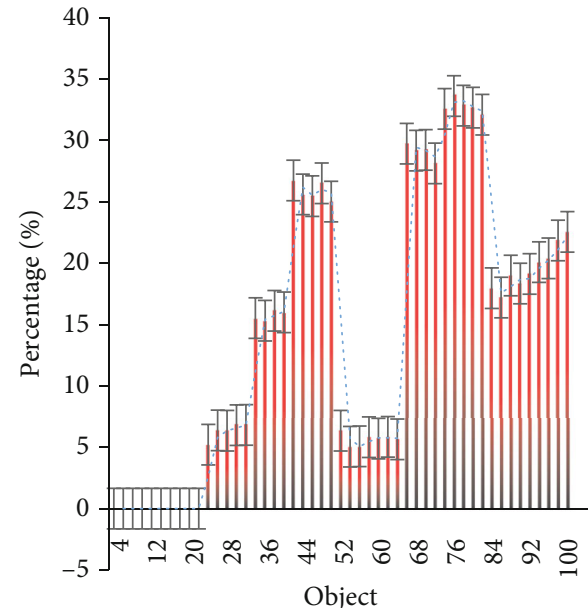

University A

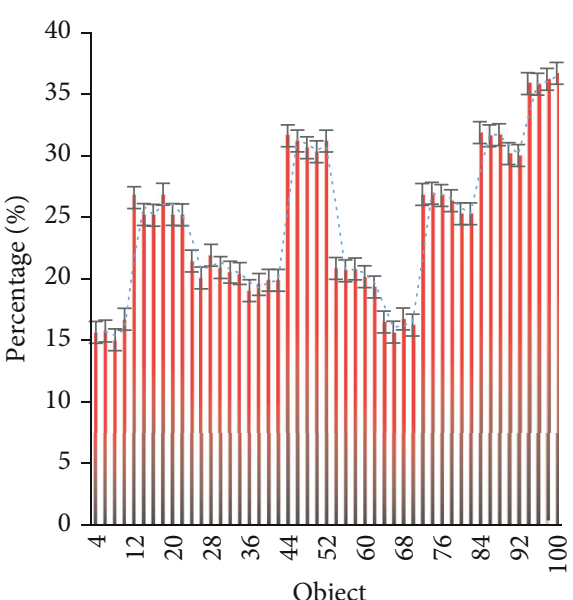

University B

FIGURE 8: Information service efficiency map based on traditional methods in two sports universities.

TABLE 4: Questionnaire on hot spots of sports information services conducted by 10 sports enthusiasts.

\begin{tabular}{lcccc}
\hline $\begin{array}{l}\text { Survey } \\
\text { object }\end{array}$ & $\begin{array}{c}\text { Use sensor } \\
\text { networks }\end{array}$ & $\begin{array}{c}\text { No sensor network } \\
\text { used }\end{array}$ & $\begin{array}{c}\text { Score } \\
\text { A }\end{array}$ & $\begin{array}{c}\text { Score } \\
\text { B }\end{array}$ \\
\hline 1 & Yes & No & 6.7 & 2.1 \\
2 & Yes & No & 7.8 & 1.8 \\
3 & Yes & No & 8.6 & 0 \\
4 & Yes & No & 8.5 & 0 \\
5 & Yes & No & 7.8 & 2.1 \\
6 & Yes & No & 8.5 & 1.5 \\
7 & No & Yes & 6.4 & 1.7 \\
8 & Yes & No & 9.8 & 1.4 \\
9 & No & Yes & 7.5 & 2.6 \\
10 & Yes & No & 5.5 & 3.1 \\
\hline
\end{tabular}

article summarizes the status quo and development problems of sports public information service industry during the social transition period by consulting a large number of sports and related interdisciplinary literature materials and serves as the theoretical basis for the research content. Next, this article investigates the efficiency of information services based on traditional methods in two sports universities, as shown in Figure 8.

As shown in Figure 8, university A's information service efficiency based on traditional methods is the highest around $33 \%$, and the lowest is around 5\%; university B's information service efficiency based on traditional methods is the highest around $35 \%$, and the lowest is around 15\%; although, universities are less efficient than universities; in general, the information services of the two schools lag behind. This article conducts a survey of 10 sports enthusiasts on the hot spots of sports information services. The survey results are shown in Table 4.

As shown in Table 4, among the 10 sports enthusiasts, 8 enthusiasts are using sports information services based on wireless sensor networks. They feel that the information on this basis is comprehensive and reliable; there are 2 sports enthusiasts who did not use the sports information service based on the wireless sensor network, but they also want to try. So, it can be known that sports information services based on wireless sensor networks should be widely used. Next, it will investigate the efficiency of information services based on the sensor network system in the other two schools, as shown in Figure 9.

As shown in Figure 9, the use of sports information services by University C and University D in 2016 is very unstable, and the highest rate will not exceed 20\%. In 2020, the use of sports information services by University C and University $\mathrm{D}$ will gradually stabilize, and the utilization rate will be higher and higher, with a maximum of $69 \%$ and a minimum of $35 \%$. It can be seen that sports information services based on wireless sensor networks are becoming more and more popular with the public. Multiple linear regression analysis can explain the quantitative relationship of dependent changes between one or more independent variables 

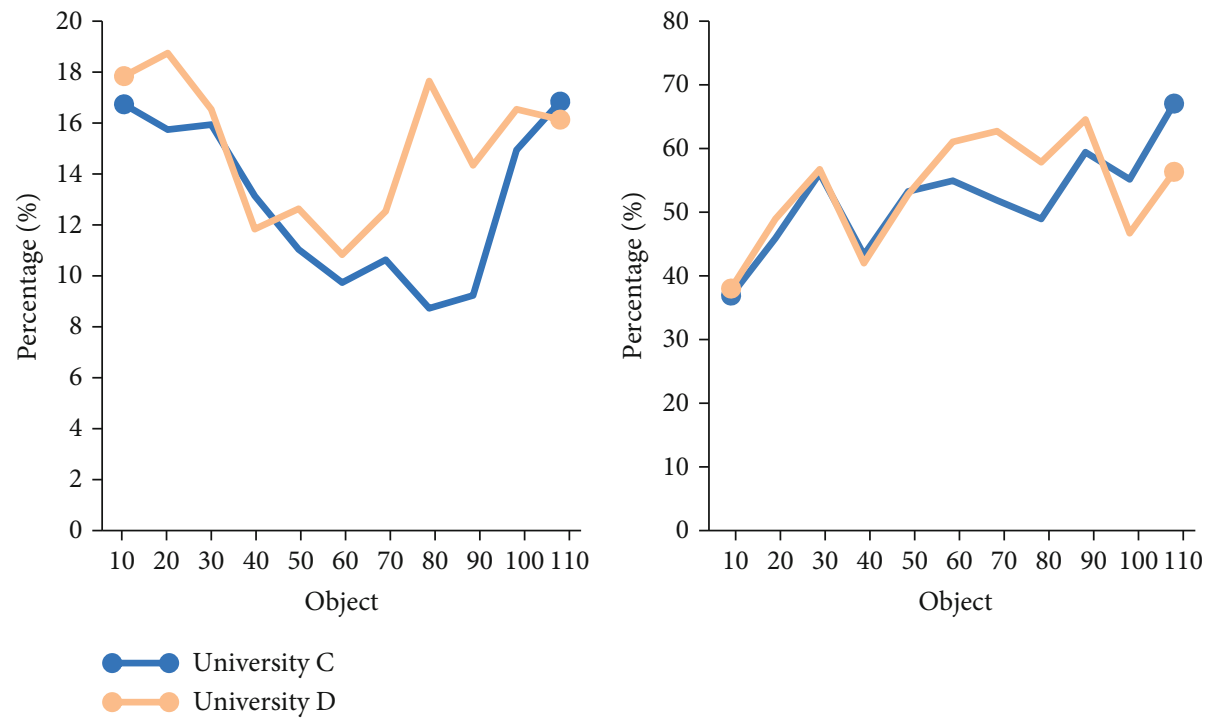

FIGURE 9: The efficiency map of two schools' information services based on the sensor network system in 2016 and 2020.

TABLE 5: Regression coefficients of the characteristics of public sports information services on the overall public satisfaction.

\begin{tabular}{|c|c|c|c|}
\hline Model & Standardization factor Beta & $t$ value & $p$ value \\
\hline (constant) & 232 & 2.321 & 0.12 \\
\hline Accuracy & 56 & 2.764 & 0.14 \\
\hline Content reliability & 231 & 1.321 & 0 \\
\hline Timeliness & 56 & 1.564 & 0 \\
\hline Openness and transparency & 76 & 3.543 & 0 \\
\hline Accessibility & 143 & 3.567 & 0 \\
\hline Patency of feed channel & 212 & 3.875 & 0 \\
\hline Effectiveness of monitoring methods & 167 & 3.543 & 0 \\
\hline
\end{tabular}

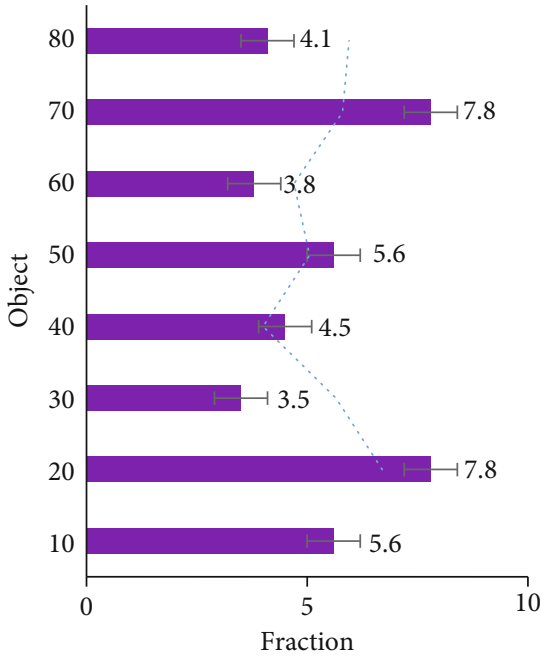

Use sensor

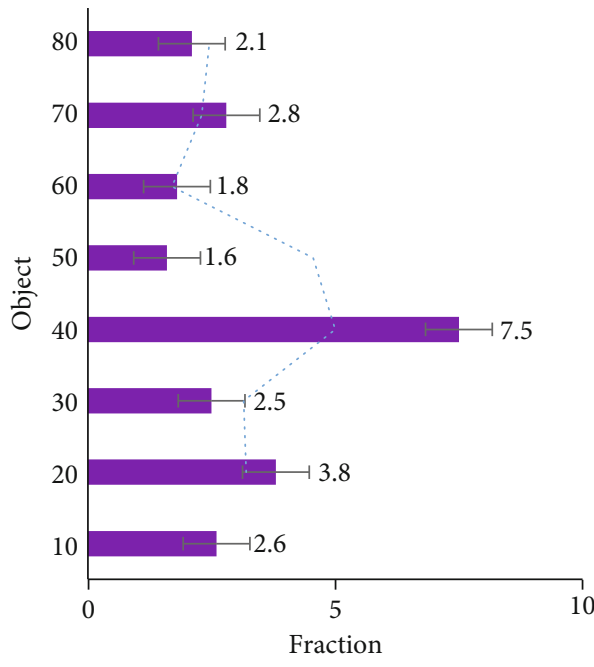

No-use sensor

FIgURE 10: Comparison chart of the scores of whether to use wireless sensor network sports services.

and the same dependent variable. Usually multiple linear regression is used to explore whether the eight independent variables will have a significant impact on the overall satis- faction of public sports information services. The results show that regression analysis is significant. It shows that the 8 independent variables are closely related to the 
dependent variable; that is, the regression line fits the sample data points to a higher degree. The summary results of regression coefficients are shown in Table 5.

As shown in Table 5, the reliability of information content, the transparency of information, the convenience of information acquisition, the smoothness of information feedback channels, and the effectiveness of information supervision methods are significantly related to the overall satisfaction of public sports information services. The effect of the accuracy of information presentation and the timeliness of information release on the overall satisfaction of information services are not significant. The reliability of information content, the transparency of information, the convenience of information acquisition, the smoothness of information feedback channels, and the effectiveness of information supervision methods can effectively predict the overall satisfaction of the public. Under the current public service system, improve the reliability of information content, the transparency of information, the convenience of information acquisition, and the smoothness of information feedback channels. And the effectiveness of information supervision methods may improve the public's satisfaction with public sports information services to a certain extent. This article analyzes the scores of sports information services that use wireless sensor networks and the scores of sports services that do not use wireless sensor networks, as shown in Figure 10.

As shown in Figure 10, it can be known that the lowest score for sports services without the wireless sensor network is 1.6 points, and the highest score is 7.5 points. The average score is around 3.5, which is very low overall; the lowest score for sports services using the wireless sensor network is 3.5 points, the highest is 7.8 points, and the average is around 5 points. The overall score is very high. Therefore, it can be known that information using wireless sensor network sports services is more popular.

The number of sports fitness information services provided is rapidly increasing. If the scope of information release cannot be expanded within time, it will be difficult to achieve sustained and effective development. Facing this huge crisis, fitness information service providers must master effective methods to expand communication and popularization and effectively promote their position in the market. Therefore, sports and fitness information service providers need to continuously improve information coverage, attract more users' attention, and realize their own development.

The experimental significance of this paper is to analyze the $5 \mathrm{G}$ embedded sensor network system, so as to obtain the role it plays in the sports information service. Through the experiment, we can know that the sports information service plays a huge role in the sports industry, and it is also convenient Sports fans get sports information.

\section{Discussion}

This article analyzes the development of sports information services based on the $5 \mathrm{G}$ embedded sensor network system, and elaborates the related concepts of the $5 \mathrm{G}$ embedded sen- sor network system and sports information services. The related theories based on $5 \mathrm{G}$ embedded sensor network system are studied,, and the design methods of sports information service hot spots are explored, through the questionnaire survey method case to discuss the importance of sports information services to contemporary society. Finally, take the $5 \mathrm{G}$ embedded sensor network system integrated into the sports information service as an example to discuss the correlation between the two.

This paper also makes reasonable use of the Kriging algorithm based on the $5 \mathrm{G}$ embedded sensor network system. As the Kriging algorithm has become more widely used, its importance has gradually become more prominent. Many scholars have begun to match the Kriging algorithm theory with real application scenarios and propose feasible algorithms. Kriging algorithm is a mathematical operation, and according to this operation, it is known that strengthening sports information services through the Internet is an indispensable part of life.

The advantages of Kriging algorithm are linear, unbiased, small variance, and suitable for spatial analysis. So, it is very suitable for geology, meteorology, geography, cartography, etc. relative to other interpolation methods. The main disadvantage is that the response speed is very slow because he has to consider the calculation influence range, consider whether the anisotropy is not, select the variogram model, and calculate the variogram value.

Through the questionnaire survey method, this article knows that the information using wireless sensor network sports services is more popular. Therefore, combining the characteristics of the "Internet + " era and finding a new communication method to enable people to receive relevant sports information services correctly and comprehensively is the key to promoting the development of sports information services.

With the rapid development of my country's economic construction, the people have gradually realized the importance of physical exercise, and sports have become a way of life for modern people. Social subjects are an important force in the construction of my country's public sports system. The public sports services provided by social subjects are not utilitarian and adhere to the principles of voluntary and semivoluntary.

\section{Conclusions}

This article mainly starts from theoretical knowledge based on 5G embedded sensor network system and sports information service and discusses how to establish a more reliable sports information service system based on sensor network system and 5G. Based on the global optimization of Kriging algorithm and sensor network structure, it can be seen that the global optimization of Kriging algorithm is applied in the process of sports information service, which can obtain better evaluation results and provide a new idea for sports information service. Moreover, the process is faster and more accurate, which has certain guiding significance for how to develop sports information services. Based on the $5 \mathrm{G}$ embedded sensor network system, the related scientific 
fields involved are relatively extensive; so, the concept of sports information service has always been disputed. It can be seen from the experiments that the $5 \mathrm{G}$ embedded sensor network system can effectively promote the development of sports information services. The author's knowledge has not yet reached the perfect position, the author's academic theory and professional ability are relatively weak, and there are still many deficiencies. But at the same time, the author is constantly discovering and solving problems, striving to be the best.

\section{Data Availability}

No data were used to support this study.

\section{Conflicts of Interest}

The authors declare that there is no conflict of interest with any financial organizations regarding the material reported in this manuscript.

\section{Acknowledgments}

This work was supported by the School-level Academic Team Project of Wuhan Business University (2018TD011), the Wuhan University of Business Doctoral Fund projects (2021KB002), and the Application Foundation Frontier Project (2020010601012294).

\section{References}

[1] X. F. Xu, P. Sun, L. Wang et al., "Study of urban sport energy conservation information network service system," Energy Education Science and Technology, vol. 34, no. 4, pp. 343350, 2016.

[2] M. S. Howard, K. L. DiDonato, D. L. Janovick et al., "Perspectives of athletes and pharmacists on pharmacist-provided sports supplement counseling: an exploratory study," Journal of the American Pharmacists Association, vol. 58, no. 4, pp. S30-S36.e2, 2018.

[3] T. J. Cain, F. M. Cheek, J. Kupsco, L. J. Hartel, and A. Getselman, "Health sciences libraries forecasting information service trends for researchers: models applicable to all academic libraries," College \& Research Libraries, vol. 77, no. 5, pp. 595-613, 2016.

[4] Q. Guo, L. Jian-Xia, and W. Yu-Ming, "Evaluation of intelligent tourism information service capabilities: a case study of Hanzhong City," Ecological Economy, vol. 1, pp. 2-13, 2021.

[5] P. Gope and T. Hwang, "BSN-care: a secure IoT-based modern healthcare system using body sensor network," IEEE Sensors Journal, vol. 16, no. 5, pp. 1368-1376, 2016.

[6] S. Abraham and X. Li, "Design of a low-cost wireless indoor air quality sensor network system," International Journal of Wireless Information Networks, vol. 23, no. 1, pp. 57-65, 2016.

[7] M. Hammoudeh, F. Al-Fayez, H. Lloyd et al., "A wireless sensor network border monitoring system: deployment issues and routing protocols," IEEE Sensors Journal, vol. 17, no. 8, pp. 2572-2582, 2017.
[8] D. Zhang, P. Shi, W. A. Zhang, and L. Yu, "Energy-efficient distributed filtering in sensor networks: a unified switched system approach," IEEE Transactions on Cybernetics, vol. 47, no. 7, pp. 1-12, 2016.

[9] L. Dong, W. Shu, X. Li, G. Han, and W. Zou, “Three dimensional comprehensive analytical solutions for locating sources of sensor networks in unknown velocity mining system," IEEE Access, vol. 5, no. 99, pp. 11337-11351, 2017.

[10] I. Butun, P. Österberg, and H. Song, "Security of the Internet of Things: vulnerabilities, attacks, and countermeasures," IEEE Communications Surveys \& Tutorials, vol. 22, no. 1, pp. 616644, 2020.

[11] A. Mukherjee, D. K. Jain, P. Goswami, Q. Xin, L. Yang, and J. J. P. C. Rodrigues, "Back propagation neural network based cluster head identification in MIMO sensor networks for intelligent transportation systems," IEEE Access, vol. 8, no. 1, pp. 28524-28532, 2020.

[12] L. Wu, Q. Zhang, C. H. Chen, K. Guo, and D. Wang, "Deep learning techniques for community detection in social networks," IEEE Access, vol. 8, pp. 96016-96026, 2020.

[13] P. Giri, K. Ng, and W. Phillips, "Wireless sensor network system for landslide monitoring and Warning," IEEE Transactions on Instrumentation and Measurement, vol. 68, no. 4, pp. 1210-1220, 2019.

[14] N. Alsaedi, F. Hashim, A. Sali, and F. Z. Rokhani, "Detecting sybil attacks in clustered wireless sensor networks based on energy trust system (ETS)," Computer Communications, vol. 110, no. 15, pp. 75-82, 2017.

[15] W. Gao, S. Emaminejad, H. Nyein et al., "Fully integrated wearable sensor arrays for multiplexed _in situ_perspiration analysis," Nature, vol. 529, no. 7587, pp. 509-514, 2016.

[16] M. Adil, H. Song, J. Ali et al., "Enhanced AODV: A Robust Three Phase Priority-based Traffic Load Balancing Scheme for Internet of Things," IEEE Internet of Things Journal, 2021.

[17] G. M. Abdulsahib and O. I. Khalaf, "Accurate and effective data collection with minimum energy path selection in wireless sensor networks using mobile sinks," Journal of Information Technology Management, vol. 13, no. 2, pp. 139-153, 2021.

[18] W. Sun, "Research on the construction of smart tourism system based on wireless sensor network," Mathematical Problems in Engineering, vol. 2021, no. 18, pp. 1-8, 2021.

[19] B. M. Todorović and D. Samardžija, "Road lighting energysaving system based on wireless sensor network," Energy Efficiency, vol. 10, no. 1, pp. 239-247, 2017.

[20] S. Hong, "Design of air quality information service based upon geographic context information model in ISO19154," Spatial Information Research, vol. 25, no. 1, pp. 39-47, 2017.

[21] B. Han, J. Li, J. Su, and J. Cao, "Self-supported cooperative networking for emergency services in multi-hop wireless networks," IEEE Journal on Selected Areas in Communications, vol. 30, no. 2, pp. 450-457, 2012.

[22] Y. C. Kaplan, B. Karadaş, G. Küçüksolak et al., "Counselling pregnant women at the crossroads of Europe and Asia: effect of teratology information service in Turkey," International Journal of Clinical Pharmacy, vol. 39, no. 4, pp. 783-790, 2017.

[23] S. Xue, L. Xiong, S. Yang, and L. Zhao, "A self-adaptive multiview framework for multi-source information service in cloud ITS," Journal of Ambient Intelligence \& Humanized Computing, vol. 7, no. 2, pp. 205-220, 2016. 
[24] T. Oh, H. Sung, and K. D. Kwon, "Effect of the stadium occupancy rate on perceived game quality and visit intention," International Journal of Sports Marketing and Sponsorship, vol. 18, no. 2, pp. 166-179, 2017.

[25] H.-W. Park and S.-H. Kim, "Analysis of factors influencing the self-perceived health status of elderly people," Korean Journal of Sports Science, vol. 26, no. 2, pp. 1213-1225, 2017.

[26] C. K. Harrison, S. Bukstein, G. M. Botts, and S. M. Lawrence, "Female spectators as customers at National Football League games," International Journal of Sports Marketing and Sponsorship, vol. 17, no. 2, pp. 172-200, 2016.

[27] A. I. Rynarzewska, "Virtual reality: a new channel in sport consumption," Journal of Research in Interactive Marketing, vol. 12, no. 4, pp. 472-488, 2018.

[28] O. I. Khalaf, G. M. Abdulsahib, and B. M. Sabbar, "Optimization of wireless sensor network coverage using the bee algorithm," Journal of Information Science and Engineering, vol. 36, no. 2, pp. 377-386, 2020.

[29] G. Dartmann, H. Song, and A. Schmeink, Big data analytics for cyber-physical systems: machine learning for the Internet of Things, Elsevier, 2019.

[30] E. Strumbelj, "A comment on the bias of probabilities derived from betting odds and their use in measuring outcome uncertainty," Journal of Sports Economics, vol. 17, no. 1, pp. 12-26, 2016.

[31] R. Biscaia, A. Correia, M. Yoshida, A. Rosado, and J. Marôco, "The role of service quality and ticket pricing on satisfaction and behavioural intention within professional football," International Journal of Sports Marketing \& Sponsorship, vol. 14, no. 4, pp. 301-325, 2016. 